\title{
MODELOS DE ANÁLISIS DE CASOS EN ÉTICA CLÍNICA
}

\author{
Rodrigo López Barreda ${ }^{1}$
}

Resumen: Los avances de la medicina ocasionan cada vez con más frecuencia casos en los que resulta difícil identificar "lo mejor o más deseable" desde el punto de vista ético. Para solucionar dichos dilemas, se recurre a modelos de análisis de casos y a modelos de fundamentación ética. Los modelos de análisis requieren atención, porque la identificación de la información relevante para emitir un juicio ético en algunas oportunidades resulta difícil. El presente artículo busca exponer algunos modelos de análisis de casos de uso frecuente en la bioética latinoamericana y hacer un análisis comparativo de los mismos. Luego de una revisión bibliográfica, se comentan seis modelos usados en la ética clínica, constatándose la gran diversidad que existe entre ellos, así como la existencia de factores ignorados que pueden interferir en el proceso de toma de decisiones éticas.

Palabras clave: modelos de análisis de casos, ética clínica, bioética

\section{Case analysis models in clinical ethics}

\begin{abstract}
Advances in medicine cause every time with more frequency cases in which it is difficult to identify what is "the best or more accepted" from an ethical point of view. In order to solve these dilemmas, case analysis models and ethical foundation models are used. The analysis models require attention, because the identification of relevant information to issue an ethical judgment is difficult in some opportunities. This article tries to show some case analysis models of frequent use in Latin American bioethics and to make a comparative analysis of them. After a bibliographic review, six models used in clinical ethics are commented, showing the great diversity existing among them, as well as the existence of ignored factors which may interfere with the ethical decisions making process.
\end{abstract}

Key words: case analysis models, clinical ethics, bioethics

\section{Modelos de análise de casos em ética clínica}

Resumo: Os avanços da medicina ocasionam cada vez com mais frequência casos nos quais resulta difícil identificar "o melhor ou mais desejável" do ponto de vista ético. Para solucionar estes dilemas, se recorre a modelos de análise de casos e a modelos de fundamentação ética. Os modelos de análise requerem atenção, porque a identificaçáo da informação relevante para emitir um juízo ético em algumas oportunidades resulta difícil. O presente artigo busca expor alguns modelos de análise de casos de uso frequente na bioética latino-americana e fazer uma análise comparativa dos mesmos. Após uma revisão bibliográfica, se comentam seis modelos usados na ética clínica, constatando-se a grande diversidade que existe entre eles, assim como a existência de fatores ignorados que podem interferir no processo de tomada de decisóes éticas.

Palavras-chave: modelos de análise de casos, ética clínica, bioética

\footnotetext{
${ }^{1}$ División de Anestesiología y Centro de Bioética, Facultad de Medicina, Pontificia Universidad Católica de Chile, Chile Correspondencia: ralopez@uc.cl
} 


\section{Introducción}

Los actos médicos son el resultado de una serie de eventos sucesivos que abarcan desde el diagnóstico, pronóstico y tratamiento, hasta la implementación y evaluación de resultados. En cada etapa del proceso se presentan al médico múltiples alternativas de acción; su elección se fundamenta en juicios de valor, donde se opta por la alternativa que resulta "mejor". Para llegar a esta decisión se ponderan los más variados elementos, como factores técnicos, económicos, éticos, emocionales y prácticos.

Habitualmente en la práctica clínica estas decisiones se toman de manera intuitiva $(1,2)$, pero en algunos casos resulta complejo identificar "lo mejor o más deseable". Se dice entonces que se trata de un dilema ético-clínico. Bernard(1) define esta situación como "la dificultad en la toma de decisiones frente a un paciente, en cuya resolución es necesario referirse a valores o principios que especifiquen lo que debe ser hecho en oposición a lo que simplemente fue hecho o frecuentemente se hace". Constituye un problema de dos dimensiones: clínico, porque se presenta en la práctica de la medicina, y ético, porque hace referencia a la búsqueda de lo correcto o incorrecto de los actos humanos.

Si definimos la bioética como "aplicación de la ética al ámbito biomédico", debe constar de elementos teóricos que, a modo de marco conceptual, permitan definir lo "bueno" y lo "malo", entre los que se cuentan los modelos utilitarista, deontológico, personalista, entre muchos otros; se suele referir a estos elementos como los "modelos de fundamentación". Por otra parte, la bioética debe disponer también de metodologías prácticas que le permitan llevar a cabo una valoración normativa de los actos concretos en el ámbito sanitario, los que han sido denominados "modelos de análisis".

Según Diego Gracia (3): "la realidad concreta es siempre más rica que nuestros esquemas intelectuales y, por lo tanto, los desborda. De ahí que el proceso de toma de decisiones no pueda consistir en una pura ecuación matemática, sino en el análisis cuidadoso y reflexivo de los principales factores implicados", lo que pone de manifiesto la importancia del enfrentamiento práctico para la resolución de los dilemas ético-clínicos.

El presente artículo busca profundizar algunos aspectos de los modelos de análisis de casos clínicos, reconociendo la relación que tienen con los modelos de fundamentación, pero sin ahondar en ellos.

Una adecuada metodología para la descripción del dilema incorpora todos los factores que se deben examinar para emitir un adecuado juicio moral. De este modo, se busca la objetividad necesaria para la resolución de los dilemas éticoclínicos que no niega la subjetividad de los actores involucrados, sino que la incluye de una manera objetiva. Del buen uso de una metodología determinada es posible que dos personas que analizan un mismo caso puedan obtener resultados comparables; de este modo se valida a la ética como una ciencia práctica, sustentada en conceptos universales y no en posturas individuales, donde el relativismo es la norma(2).

\section{Metodología}

La búsqueda bibliográfica se efectuó utilizando los buscadores Medline y ProQuest, usando como palabras claves "modelo de análisis de casos", "ética clínica", "bioética” y "ética de la administración de empresas", tanto en castellano como en inglés. La busqueda fue limitada al periodo entre los ańos 1990 y 2010. Los modelos más citados en la literatura encontrada son los finalmente incluidos en la presente revisión.

\section{Resultados}

\section{Clasificación general}

En términos generales, es posible clasificar los modelos de análisis de dilemas éticos en dos grupos: normativos y descriptivos(4).

1) Los modelos normativos surgen a partir de la moral tradicional y pueden ser vistos como la aplicación de un modelo de fundamentación determinado a los casos de la vida cotidiana. "Las estrategias o métodos éticos clínicos recientes no son completamente nuevos, sino que en el curso de la historia han sido precedidos por otras versiones de cómo llegar a conclusiones defendibles 
que se encuentran en la ética religiosa. La teología moral del catolicismo, en particular, se interesó en estas estrategias para orientar las decisiones de los directores espirituales o para usar en la confesión" (2). Se describe que, debido a su origen, estos modelos son más efectivos en establecer lo correcto o incorrecto de una determinada conducta(4); sin embargo, la descripción que obtienen de la situación puede ser insuficiente, con el riesgo de no incluir en el análisis todos los elementos a considerar.

La gran mayoría de los modelos utilizados en la ética clínica se pueden clasificar en este grupo.

Es posible reconocer en este tipo de modelos dos etapas: una fase descriptiva, en que se recopilan datos sobre el problema a analizar, y una fase de juicio, en que se aplican las normas o un sistema de fundamentación ética a la situación particular, que ahora se comprende mejor gracias a la información recopilada en la fase anterior.

Frecuentemente, un escenario que inicialmente parece ser de difícil solución resulta ser solo un problema aparente, luego de ser caracterizado de una manera más completa. Estas dos fases están muy relacionadas, porque el juicio al que se llegue al aplicar las normas depende de la información recopilada, pero también la búsqueda de datos se verá influida por el modelo de fundamentación ética con cual que se trabaja. Así, los modelos con una fundamentación utilitarista hacen mayor hincapié en la exposición de las consecuencias de las alternativas de acción durante la fase descriptiva; en un modelo deontológico se describe detalladamente del acto en sí, limitando la información sobre las consecuencias y el contexto particular.

2) Por otra parte, los modelos descriptivos provienen de disciplinas que estudian el comportamiento humano, como la psicología, y se basan principalmente en teorías y trabajos experimentales sobre el proceso de toma de decisiones, identificando los factores que intervienen en la resolución del conflicto. Aunque originariamente no estaban destinados a evaluar la moralidad de los actos humanos, se argumenta que "el procedimiento ofrece varios beneficios (...) nos puede ayudar a clarificar nuestros valores, esto es, las preferencias entre los posibles resultados a través de los cua- les juzgamos cuál podría ser la mejor decisión. El análisis de decisión también puede ser usado para aportar racionalidad y lógica a nuestras decisiones intuitivas..." (4). Es decir, estos modelos obtienen una mejor comprensión del proceso de toma de decisiones, por lo que pueden ser de utilidad en problemas como conflictos de interés, evaluación y promoción de los valores institucionales y en planificación de programas de educación.

Un claro prototipo de estos modelos es el propuesto por Thomas Jones(5). Este modelo resalta la importancia de reconocer el dilema moral, sin pretender identificar la mejor solución para el mismo. El concepto de "intensidad moral" se relaciona de manera directamente proporcional con la probabilidad de identificar un problema ético en una situación determinada $(5,6)$. Las variables a analizar son las siguientes: probabilidad (certeza de la relación causa-efecto entre la acción y la consecuencia negativa), proximidad (distancia física, social, cultural o psicológica que separa a quien toma la decisión de las consecuencias), consenso social (grado de acuerdo social al calificar a una consecuencia de la acción como incorrecta o negativa), inmediatez temporal (proximidad en el tiempo), concentración (el inverso de las personas afectadas por una acción de magnitud determinada), magnitud (sumatoria de las consecuencias negativas). Un acto con alta probabilidad de generar un dańo significativo e inmediato a varias personas próximas al agente, tendrá una gran intensidad moral, y resulta altamente improbable que no sea reconocido como un dilema ético $(5,6)$.

Debido a la gran heterogeneidad de los modelos descriptivos, es difícil plantear una estructura común; sin embargo, el trabajo de James Rest(7) aporta los conceptos necesarios para establecer un método que permita comparar estas metodologías. Este autor caracteriza los procesos psicológicos de un individuo que se enfrenta a un dilema moral, identificando cuatro fases $(7,8)$.

- Sensibilidad moral, que incluye la interpretación de la situación y el reconocimiento del modo en que las alternativas de acción pueden afectar a los involucrados; es decir, las razones por las que la situación en sí representa un problema para el sujeto. 
- Juicio moral, es decir, el proceso deliberativo que debe efectuarse, en que se refleja la integración de valores e ideales con las circunstancias pertinentes.

- Motivación moral, en que los agentes se identifican y comprometen con la propuesta y aceptan la responsabilidad por las consecuencias de la misma, es decir "desean" llevarla a cabo. En último término, es reflejo de las razones que tienen las personas para comportarse correctamente.

- Para la realización de la alternativa elegida en el Juicio moral se recurre al Carácter moral, que incluye habilidades y estrategias necesarias para una adecuada ejecución de la decisión (comunicación, autocontrol, empatía, honestidad).

Estas cuatro fases del modelo propuesto por Rest no deben ser interpretadas como etapas secuenciales, sino como procesos que pueden ocurrir en paralelo(8).

Estos conceptos se suporponen con el concepto de Conciencia moral: mientras el último se entiende en filosofía como la aplicación del conocimiento moral a cada caso particular(9), el trabajo de Rest proviene de la psicología. La Conciencia moral abarca muchos aspectos de sensibilidad, juicio y carácter, pero los aspectos afectivos propios de las decisiones éticas se tratan en mayor medida con constructos como la Motivación moral.

La clasificación de los modelos de análisis se resume en la tabla 1.

\section{Modelos de la ética clínica}

\section{1) Casuística}

La casuística se define como "la interpretación de los problemas morales, empleando procedimientos del razonamiento basado en paradigmas y analogías, que conduce a la formulación de una opinión calificada sobre la existencia y el rigor de ciertas obligaciones particulares, expresadas en términos de normas o máximas generales, pero no universales ni invariables, dado que se aplican con certeza únicamente en las condiciones características del agente y las circunstancias de acción" (2). Este es uno de los modelos para la resolución de los dilemas morales de más larga data, y se originó en el estoicismo, entre los siglos III a.C. y II d.C., y floreció entre los siglos XV y XVI. Debido a su simpleza, llegó a ser uno de los modelos más utilizados en la bioética actual $(2,10)$.

De acuerdo con este método, los elementos del acto moral —objeto, fin y circunstancias - son la fuente para la calificación moral del mismo, por lo tanto deben ser analizados conjuntamente sin poder aislar el acto de los factores que lo rodean. La descripción detallada de estos elementos constituiría un "caso" sobre el cual se realizan los juicios morales. El patrón para evaluar la conducta a seguir son las "máximas", entendidas como normas generales de acción - por ejemplo, "el médico no debe dañar a sus pacientes", "a los pacientes no se les deben imponer los tratamientos". Como su aplicación se efectúa en casos definidos, no es posible establecer la validez universal de ninguna máxima.

Tabla 1: Clasificación de modelos de análisis de casos

\begin{tabular}{|l|l|l|}
\hline Modelos normativos & & Modelos descriptivos \\
\hline $\begin{array}{l}\text { Aplicación de un modelo de } \\
\text { fundamentación ética }\end{array}$ & \multirow{2}{*}{ Objetivo } & $\begin{array}{l}\text { Descripción de elementos y del proceso de toma } \\
\text { de decisión }\end{array}$ \\
\hline Teórica & Fundamentación & Teórico y empírica \\
\hline Descriptiva & \multirow{5}{*}{ Fases } & Sensibilidad \\
\cline { 1 - 1 } & & Juicio \\
\cline { 3 - 3 } & & Motivación \\
\cline { 3 - 3 } & & Carácter \\
\hline Elección de un curso de acción & Utilidad & Identificación de elementos "encubiertos" \\
\hline
\end{tabular}


Es posible que varias máximas se contradigan en un mismo caso (comunicación de malas noticias: "el médico debe decir la verdad a sus pacientes" y "el médico debe procurar el bienestar de sus pacientes"), por lo que en el análisis se debe determinar cuál máxima es la que prima. En la casuística clásica existen "casos-paradigma” en los que está claramente establecido cuáles máximas prevalecen en las circunstancias especificadas, por lo que si por analogía una situación se homologa a uno de estos paradigmas, las mismas máximas deben prevalecer.

Albert Jonsen(10) realiza una sistematización de dicho modelo, que se muestra a continuación.

El caso: hechos (personas, tiempo, lugar, acciones, etc); opiniones; máximas; valores.

Presentación del caso: indicaciones médicas (diagnóstico y pronóstico, objetivos terapéuticos, eficacia/ineficacia, utilidad/futilidad); preferencias del paciente (principio de autonomía, capacidad de elección/competencia, consentimiento informado, rechazo, decisiones de sustitución, directrices previas); calidad de vida; factores socioeconómicos (familia, costos, distribución de recursos, investigación, confidencialidad, protección de otros); otros tópicos que contribuyan a caracterizar el caso y el problema.

Resolviendo el caso: máximas más importantes y principios involucrados; taxonomía (clasificación y analogía de casos similares); soluciones probables y razonables.

Debido a su estructura, este modelo puede clasificarse dentro de los normativos: los puntos 1 y 2 corresponden a la fase descriptiva; el punto 3 es la fase de juicio, en el que se aprecia la relevancia tanto de las máximas como de la analogía para identificar la alternativa correcta.

\section{2) Modelo propuesto por Thomasma y Pellegrino}

Durante las décadas de 1960 y 1970 comenzó a difundirse el concepto de ética médica como una entidad particular de importancia en la resolución de dilemas ético-clínicos. David Thomasma y Edmund Pellegrino fueron pioneros en proporcionar a los clínicos una metodología para abor- dar estos dilemas(11). En el transcurso de los años el modelo sufrió algunas modificaciones, pero básicamente consta de cinco fases que se muestran a continuación.

- Descripción de los hechos médicos del caso.

- Describir los valores (metas, intereses de todas las partes involucradas en el caso: médicos, pacientes, personal, hospital).

- Indicar cuál es el principal conflicto de valores.

- Determinar las posibles medidas que podrían proteger el mayor número posible de valores en el caso.

- Defender la decisión tomada.

Esta metodología resulta familiar a los médicos clínicos que utilizan un razonamiento similar en la resolución de los problemas técnicos. Es un buen ejemplo de un modelo normativo, con una fase descriptiva presente en los puntos 1,2 y 3, y una fase de juicio en los puntos 4 y 5 . La autonomía del paciente, un elemento central de la bioética anglosajona, se encuentra presente de manera poco explícita, probablemente por ser un método creado previo al auge de la autodeterminación.

\section{3) Modelo propuesto por James Drane}

Los cinco pasos del modelo anteriormente enunciado son esquematizados en cuatro etapas por el norteamericano James Drane(2), organizándolos según fases temporales o "niveles discursivos". En el primero de estos niveles se describen los elementos que conforman el caso. Luego se realiza el proceso de deliberación, el que se encuentra dividido en análisis racional y volitivo, y que culmina con la toma de decisión. Por ultimo, presenta una fase pública, en la cual se preparan los argumentos para comunicar la decisión y validarla como la mejor alternativa. En cada una de estas etapas integra un mayor número de consideraciones, para obtener una descripción acabada del problema y una deliberación racional que incorpore elementos afectivos. El modelo propuesto se sintetiza del siguiente modo:

Fase expositiva - Guía para señalar los hechos per- 
tinentes: factores médicos (diagnóstico, pronóstico, opciones terapéuticas, incertidumbre, etc.); factores personales (intereses, deseos, sentimientos, pensamientos y preferencias del paciente, los médicos, el personal, los administradores del hospital y la sociedad); factores socioeconómicos (costos económicos en sentido amplio).

Fase racional - Guía para el análisis de los datos pertinentes: categorías relacionadas con la ética médica (consentimiento, confidencialidad, experimentación, eutanasia, etc.); principios y máximas (beneficencia, autonomía, respeto, verdad, fidelidad, santidad de la vida, justicia y otras normas generales o específicas); decisiones de índole jurídica y códigos profesionales (casos paradigmáticos, códigos de ética profesional, generales y locales).

Fase volitiva - El paso de los hechos y la reflexión a la adopción de decisiones: ordenamiento de los beneficios (enumeración de los intereses comprometidos y ordenamiento según precedencia); ordenamiento de los principios (enumeración de principios comprometidos y ordenamiento según creencias personales y/o compromisos profesionales); adopción de una decisión.

Fase pública - Preparación para el escrutinio público y la defensa de las decisiones: explicitar las hipótesis y tomar conciencia de los factores subjetivos y de las creencias fundamentales; establecer una correlación entre las razones y los sentimientos (esforzarse por aplicar los principios, las máximas y las normas en forma coherente); organizar los argumentos para el discurso público.

Este modelo podría ser clasificado junto a los normativos, porque se vislumbra claramente la fase descriptiva en los puntos 1,2 y 3 , y la fase de juicio al final del punto 3. No obstante lo anterior, es necesario destacar que incorpora una fase volitiva que incluye elementos no considerados usualmente por este tipo de modelos. Por otra parte, llama la atención que se recabe información sobre "los intereses, deseos, sentimientos, pensamientos y preferencias... los administradores del hospital y la sociedad" y sobre "decisiones de índole jurídica y códigos profesionales - casos Paradigmáticos", probablemente en respuesta a la medicina norteamericana, en que la "salud administrada" y la legalización de los casos médicos son fenómenos cada vez más presentes(12). En ese mismo sentido, es destacable la consideración hecha a la "incertidumbre relacionada con la comprensión científica en el ejercicio de la medicina", mucho más presente en la bioética europea que en la bioética norteamericana.

\section{4) Modelo propuesto por Diego Gracia}

Uno de los modelos normativos más influyentes en la bioética hispanoamericana es el propuesto por Diego Gracia. Haciendo eco a lo propuesto por Richard Hare en 1991, Gracia plantea que el análisis de los problemas morales ocurre a dos niveles: una etapa prerracional, en que se toman en cuenta aprehensiones morales básicas, y una etapa racional, en que se evalúan las consecuencias de los posibles cursos de acción. La etapa prerracional, o momento deontológico, se expresa a través de deberes o normas prima facie, que son identificados operacionalmente con los principios enunciados por Beauchamp y Childress, pero ordenados jerárquicamente en dos grupos: nivel 1, no-maleficencia y justicia; nivel 2, beneficencia y autonomía $(3,13)$. La razón de este ordenamiento radica en que, según Gracia, los de primer nivel son los mínimos exigibles a todas las personas, socialmente aceptados y en general expresados en las leyes; los de segundo nivel, en cambio, son loables pero no exigibles, y reflejan los ideales y creencias individuales. La metodología propuesta por $\operatorname{Gracia}(3,13)$ se sistematiza a continuación.

El sistema de referencia moral: premisa ontológica (el hombre es persona, y en tanto que tal tiene dignidad y no precio); premisa ética (en tanto que personas, todos los hombres son iguales y merecen igual consideración y respeto).

El momento deontológico del juicio moral: nivel 1 (no-maleficencia y justicia); nivel 2 (beneficencia y autonomía).

El momento teleológico del juicio moral: evaluación de las consecuencias objetivas (de nivel 1); evaluación de las consecuencias subjetivas (de nivel 2).

El juicio moral: contraste del caso con la regla (paso 2); evaluación de las consecuencias del acto 
(paso 3), para ver si es necesario hacer una excepción a la regla; contraste de la decisión tomada con el sistema de referencia (paso 1); toma de la decisión final.

De acuedo con la estructura y el objetivo de este modelo, parece pertinente incluirlo dentro de los normativos, ya que recopila información (etapas 1,2 y 3) y proporciona una metodología clara para llegar a una respuesta (etapa 4). Según este modelo, la "mentira piadosa" podría ser justificable: en el momento deontológico resulta incorrecta porque falta a los principios de no-maleficencia y autonomía, pero debido a las eventuales consecuencias de entregar información veraz, es posible que resulte apropiado hacer una "excepción a la regla".

\section{5) Modelo propuesto por Bernard Lo}

El mismo médico que acuña el término "dilema ético-clínico" propone un modelo para ayudar en la resolución de los mismos. Basado en la práctica habitual, el método es bastante flexible ya que permite a "un médico experimentado modificar la aproximación general"(14) y que se describe del siguiente modo:

Recolectar la información: ¿cuál es la situación médica? Si el paciente es competente, ¡cuáles son sus preferencias? Si el paciente no es competente, ¿existe una "voluntad anticipada"? Si el paciente no es competente, ¿quién lo representa? ¿Cuál es la opinión del resto del equipo de salud? ¿Existen dificultades prácticas en la resolución del caso?

Clarificar el conflicto ético: ¿qué dilemas éticos son pertinentes de enfrentar? Referencia a guías éticas usadas habitualmente. ¿Cuáles son los argumentos a favor y en contra de cada alternativa?

Resolver el dilema: reunir al equipo de salud con el paciente (o su representante). Exponer las alternativas de acción. Negociar una decisión mutuamente aceptable.

Este modelo puede ser clasificado junto a los normativos, con quienes comparte una finalidad y estructura similar. Destaca en esta propuesta el reconocimiento del personal de salud como sujetos independientes que, por una parte, son respon- sables de sus actos y, por lo tanto, deben estar de acuerdo con la solución elegida para permitir su implementación, y por otra tienen una opinión que merece ser escuchada.

\section{6) Modelo propuesto por Verónica Anguita}

Este modelo responde a dos problemas de los comités de bioética actuales: la falta de una metodología estandarizada para la resolución de estos dilemas, y "la falta de formación específica en bioética, así como la falta de tiempo real para formarse y la urgencia de conseguir la propuesta de decisiones adecuadas y aceptables para todos"(15).

Propone un método basado en la deliberación, entendida como "una forma de comunicación donde se manifiesta la expresión del yo, a la vez que se siente que el otro respeta y escucha"(15), lo que resalta la importancia de la subjetividad de quienes se ven enfrentados al proceso de toma de decisiones.

Este modelo plantea que las personas recurren a la experiencia personal frente a situaciones conflictivas, incluso de manera inconsciente, como una forma de comprender y dimensionar mejor las variables que intervienen en el problema en cuestión; luego de este ejercicio se interpretan mejor las posibles alternativas y las consecuencias derivadas de las mismas. Al mismo tiempo, el modelo reconoce que el dilema ético-clínico se presenta a un individuo (el profesional de salud), quien comienza este proceso de manera personal. Cuando encontrar una solución satisfactoria resulte difícil, recurre a un comité de bioética, en el que de manera grupal deberá iniciar el mismo proceso antes enunciado. Luego de la propuesta final del comité, el mismo individuo a quien se le presentó el problema es el encargado de implementarla, produciéndose un nuevo momento personal. Las distintas fases de este modelo se sistematizan a continuación.

Proceso personal: experiencia; interpretación; juicio; propuesta.

Proceso grupal: experiencia (investigación: datos y aclaraciones; conocimiento: relación de información y aceptación de respuesta subjetiva); 
interpretación (historia: situaciones similares); dialéctica: comparación con casos anteriores; evaluación de distintos escenarios; formación de conciencia (creencias, valores, etc.); compromiso con el caso; juicio (fundamentos: identificación de valores y criterios; experiencias previas: confrontación con enseñanzas autorizadas); propuesta (sistemática: referencia a los puntos analizados anteriormente; comunicación: de la solución y sus fundamentos).

Proceso personal: acción; auto-evaluación (incluye la adquisición de habilidades para futuros casos).

Debido a la estructura y fases de este modelo, no es evidente calificarlo dentro de los normativos, sino que parece más bien ser de tipo descriptivo, ya que se centra en torno a los distintos proceso a los que se ve la persona que enfrenta la situación. Si bien puede parecer una metodología eminentemente subjetiva, la fase de juicio grupal hace eco a este asunto al recurrir a las "enseñanzas autorizadas", buscando objetividad en la resolución de dilemas ético-clínicos.

\section{Discusión}

Aunque no es objetivo de este artículo ahondar en los modelos de fundamentación ética, cabe destacar la relación entre algunos de estos modelos de análisis con distintos marcos de fundamentación teórica. Por ejemplo, los modelos de Lo y de Anguita, que recurren a la negociación colectiva como una manera de resolver el dilema, hacen suponer que el sistema de fundamentación ético en estos caso es la ética de consensos. El modelo propuesto por Thomasma muestra la influencia de la ética de consensos en la descripción del caso, al incorporar la visión "de todas las partes involucradas", y algunos aspectos que asemejan fundamentación utilitarista al momento del juicio moral, ya que requiere "determinar las posibles medidas que podrían proteger el mayor número posible de valores en el caso"(11). El modelo propuesto por Gracia apela directamente a los modelos deontólógicos y utilitarista; al primero durante el "momento deontológico" y al segundo durante la "evaluación de las consecuencias" $(3,13)$. Resulta más difícil establecer dichas asociaciones con modelos como la casuística y el modelo de
Drane. Como fue comentado previamente, la casuística tiene una larga data, por lo que antecede a muchos de los modelos de fundamentación actualmente en uso; el modelo de Drane se basa fuertemente en la casuística, lo que se percibe al referirse explícitamente a "principios y máximas" y a "decisiones de índole jurídica y códigos profesionales" (2).

En cuanto a la clasificación como descriptivos y normativos, la gran mayoría de estos modelos pueden ser fácilmente categorizados como normativos: la finalidad de prácticamente todos es identificar el curso de acción más apropiado a implentar, recurriendo a la descripción del caso y al juicio moral. Sin embargo, los modelos de Drane y Anguita incorporan algunos elementos extras, que serán comentados más adelante.

Siguiendo esta clasificación, no resulta complejo hacer un análisis comparativo utilizando las fases de los modelos normativos. La fase descriptiva está presente en todos los modelos. Muchos comienzan por describir los hechos concretos que generan el conflicto — salvo Beauchamp y Gracia, quienes primero definen el sistema de valores o código que se utilizará en el análisis posterior. Luego de presentados los hechos, así como la información adicional, en casi todos los modelos se explicita cuál es el conflicto y cuáles son los valores en juego. En la fase de juicio es donde se toma la decisión final. Algunos modelos, como los de Thomasma, Drane y Anguita, incluyen una etapa posterior en que se reevalúa la decisión, ya sea por la misma persona que la tomó o por la sociedad.

Sin embargo, los modelos normativos pueden excluir información relevante, al momento de la toma de decisiones, referente a la subjetividad de quien se ve enfrentado a dicho proceso. Los modelos descriptivos, por su parte, aunque no tienen como objetivo proporcionar una decisión adecuada, al centrarse en dichos aspectos, pueden ser de utilidad al proporcionar información sobre estos aspectos no considerados. De este modo, resulta interesante hacer un análisis comparativo entre los modelos normativos y descriptivos, buscando los posibles puntos convergentes, así como los que podrían ser de utilidad a los modelos normativos para obtener una mejor descripción de la situación. 
Al hacer un paralelo en las fases de los dos tipos de modelos de análisis, la etapa descriptiva de los modelos normativos se podría relacionar con la Sensibilidad moral, porque hace referencia a la situación que genera el conflicto. Sin embargo, mientras los modelos normativos buscan identificar cuáles de los valores en juego se ven representados en las distintas alternativas de acción, la sensibilidad moral de los modelos descriptivos incluye elementos de la subjetividad del evaluador, es decir, las razones por las que le resulta difícil tomar la decisión. La Motivación moral también se podría encontrar implícitamente incorporada en la fase descriptiva, cuando se hace referencia al sistema de valores en juego (por ejemplo, en el modelo de Gracia); pero hacer referencia al marco valórico no refleja necesariamente las razones por las que la persona que toma la decisión se compromete con dicho marco, por lo que los elementos subjetivos quedan, una vez más, excluídos. El Juicio moral de los modelos descriptivos se asemaja bastante a la fase de juicio de los modelos normativos, sin existir elementos significativos que las diferencien. Por último, elementos referentes al Carácter moral se encuentra totalmente ausente en los modelos de análisis normativos.

Al hacer el análisis de los modelos expuestos utilizando las fases de los modelos descriptivos, una de las primeras cosas que es posible constatar es la poca representación en los modelos normativos de la Sensibilidad moral. El modelo propuesto por Anguita tiene un abordaje general, apelando primero a la experiencia del sujeto individual y luego al diálogo que surge entre los miembros del comité de ética.

Como se dijo, la Motivación moral se refiere a las razones que tiene la persona para adscribir a un comportamiento moralmente correcto y que determinan una vinculación con la decisión tomada. Puede reconocerse en algunos elementos de la fase volitiva del modelo de Drane, que incorpora la subjetividad de quien se enfrenta al problema. La propuesta de Thomasma-Pellegrino indirectamente hace referencia a la Motivación al incorporar una "defensa a la decisión tomada": para defender la decisión, la persona que realiza la elección debe comprometerse y aceptar las responsabilidades que se deriven de la misma.
Por último, el Carácter moral se encuentra expresado en los modelos bioéticos de Drane y Anguita; el primero al "establecer una correlación entre las razones y los sentimientos"(2), apela al desarrollo de un comportamiento coherente por parte del sujeto. Por su parte, Anguita explicita dentro de su modelo la adquisición de habilidades que serán de utilidad en futuros eventos similares. Se aprecia así la estrecha relación que tiene para estos autores la acción con la persona que ejecuta el acto: por un lado debe existir una concordancia entre los sentimientos de la persona con los actos concretos y, por otro, al realizar los actos la persona adquiere nuevas potencialidades.

Como es posible ver, los modelos de análisis de casos clínicos son bastante diversos. Si bien el tratamiento a la descripción del problema resulta comparable, existen elementos que son acogidos por algunos y dejados de lado por otros.

Probablemente resulte discutible si de la incorporación de estos elementos al análisis de casos clínicos se obtiene más información relevante para la toma de decisión. Sin embargo, estos factores pueden interferir en el proceso de toma de decisión y ameritan un reconocimiento en los modelos utilizados.

\section{Agradecimientos}

El autor de este articulo agradece a Ángela Arenas Massa y a Paula Bedregal García por revisar y corregir este artículo, proporcionando múltiples comentarios que indudablemente enriquecieron el trabajo final. 


\section{Referencias}

1. Lo B, Schroeder S. Frequency of Ethical Dilemmas in a Medical Inpatient Service. Archives of Internal Medicine 1981; 141: 1062-1066.

2. Drane J. Métodos de Ética Clínica. Boletín de la Oficina Sanitaria Panamericana 1990; 108: 415-425.

3. Gracia D. Bioética para clínicos. Medicina Clinica, 2001;117:18-23.

4. Cottone R, Claus R. Ethical Decision-Making Models: A Review of the Literature. Journal of Counseling and Development 2000; 78: 275-283.

5. Jones T. Ethical Decision Making by Individuals in Organizations: An Issue-Contingent Model. Academy of Management Review 1991; 16: 231-248.

6. Whitter N, Williams S, Dewett T. Evaluating ethical decision-making models: a review and application. Social and Business Review 2006; 1: 235-247.

7. Rest J. Development in judging moral issues. Minneapolis, MN: University of Minnesota Press; 1979.

8. Walker L. The Model and the Measure: an appraisal of the Minnesota approach to moral development. Journal of Moral Education 2002; 31: 343-367.

9. Vargas J. Formación de la conciencia moral: referentes conceptuales. Revista Educación y Desarrollo Moral 2009; 3 : 108-128.

10. Jonsen A, Siegler M, Winslade W. Clinical ethics: a practical approach to ethical decisions in clinical medicine. New York, NY: McGraw-Hill, Health Professions Division; 1998.

11. Thomasma D, Marshal P. Clinical Medical Ethics cases and readings. Lanham, MD: University Press of America; 1995.

12. Duska R. Managed Care Insurance: Some Ethical Considerations. Journal of Financial Service Professionals 2000; 54: 68-77

13. Gracia D. Bioética clínica. Bogotá: El Búho; 1998.

14. Lo B. Resolving ethical dilemas: a guide for clinicians. Baltimore, MD: Williams \& Wilkins; 1995.

15. Anguita V. La presentación de casos clínicos al Comité de Ética Hospitalaria. En: León Correa FJ, Simó RM, Schmidt L, Anguita V, (coords). Análisis de casos ético clínicos. Santiago de Chile: Felaibe; 2013: 14-24.

Recibido: 8 de enero de 2014

Aceptado: 14 de marzo de 2014 